\title{
Protein phosphorylation in mammalian spermatozoa
}

\author{
Françoise Urner ${ }^{1}$ and Denny Sakkas ${ }^{2}$ \\ ${ }^{1}$ Clinic of Sterility, Department of Obstetrics and Gynecology, University Hospital of Geneva, \\ 1211 Geneva 14, Switzerland; and ${ }^{2}$ Department of Obstetrics and Gynecology, Yale University \\ School of Medicine, New Haven, CT 06520-8063, USA
}

\begin{abstract}
Spermatozoa undergo a series of changes before and during egg binding to acquire the ability to fuse with the oocyte. These priming events are regulated by the activation of compartmentalized intracellular signalling pathways, which control the phosphorylation status of sperm proteins. Increased protein tyrosine phosphorylation is associated with capacitation, hyperactivated motility, zona pellucida binding, acrosome reaction and sperm-oocyte binding and fusion. The main tyrosine phosphorylated proteins during the course of capacitation and fertilization are localized to the flagellum, although tyrosine phosphorylation of less abundant proteins may also be regulated in the sperm head. Spermatozoa bound to the zona pellucida and fusing with the oocyte plasma membrane are characterized by a tyrosine phosphorylated flagellum. Protein phosphorylation in the flagellum is linked to hyperactivated motility in spermatozoa, but may also regulate additional functions involved in sperm-oocyte fusion. Factors involved in the appearance of phosphorylation more likely arise from the milieu surrounding the spermatozoa, but their uptake and processing are likely to be regulated differentially at specific steps within the female genital tract and during penetration of the egg vestments. One of these factors is glucose, the metabolic products of which (ATP and NADPH) appear to participate in signalling pathways by supporting a precise onset of tyrosine phosphorylation in the sperm flagellum leading to successful fertilization.
\end{abstract}

Protein phosphorylation is a post-translational modification of proteins that allows the cell to control various cellular processes. In eukaryotic cells, most phosphorylation occurs on serine or threonine residues and to a lesser extent on tyrosine residues. The phosphorylation state of phosphoproteins is controlled by the activity of protein kinases and phosphatases.

Mature spermatozoa have their own particular idiosyncrasies as highly specialized cells. They are highly compartmentalized, transcriptionally inactive and unable to synthesize new proteins. Therefore, it could be argued that the reliance of mature spermatozoa on protein phosphorylation as a means of altering their function is greater than in many other types of cell. During fertilization, sperm function is regulated by the activation of intracellular signalling systems that control protein phosphorylation. Serine/threonine and tyrosine phosphorylation occur in spermatozoa, but only a few phosphorylated proteins have been identified. Although cAMP-dependent protein kinase A plays a central role in sperm function and has been studied in detail (Visconti

Email: francoise.urner@hcuge.ch and Kopf, 1998), knowledge about tyrosine kinases and other seronine/threonine kinases remains limited.

The processes regulated by protein phosphorylation include capacitation, hyperactivated motility and the acrosome reaction, all of which are required for spermatozoa to reach and fuse with the oocyte. In general, protein phosphorylation in mammalian spermatozoa has been studied by examining phosphorylated proteins in sperm populations during capacitation and after exposure to inducers of the acrosome reaction using western blot analysis. This approach has led to the accumulation of information on the molecular weight of the phosphorylated proteins and their regulation during capacitation and acrosome reaction. Phosphorylation in individual spermatozoa has also been examined by immunocytochemistry, which has revealed compartmentalization of the phosphoproteins in individual spermatozoa and heterogeneity in sperm populations. Knowledge of the phosphorylation pattern in fertilizing spermatozoa and its regulation during gamete interaction remains one of the most important issues in this area of investigation. This review summarizes the accumulated knowledge concerning regulation of protein tyrosine phosphorylation in the 


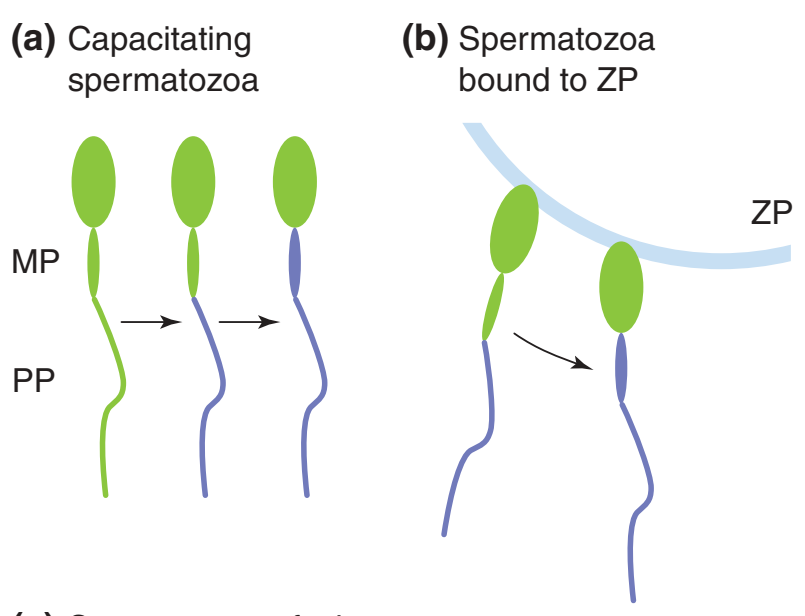

(c) Sperm-oocyte fusion

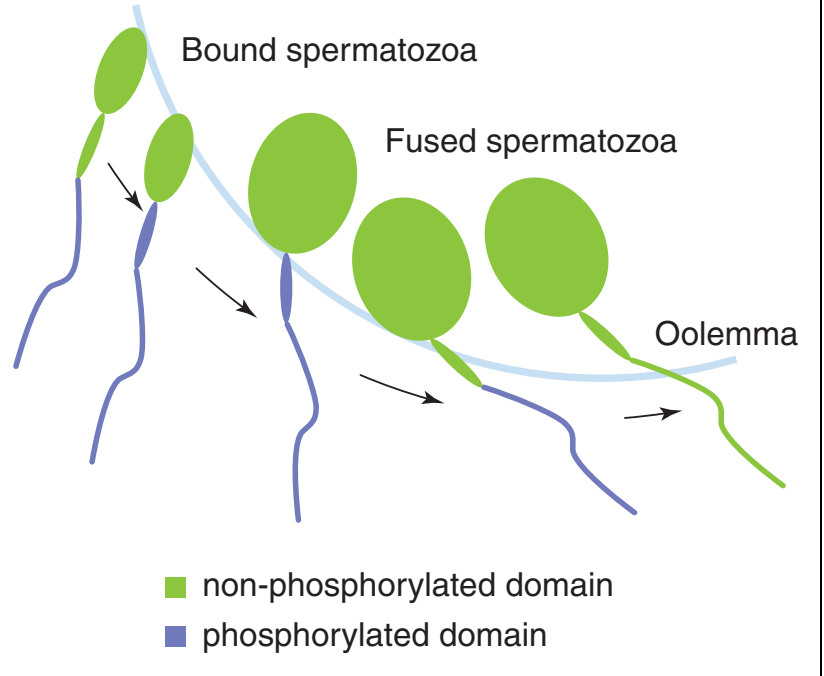

Fig. 1. Protein tyrosine phosphorylation localized to the mouse sperm flagellum during capacitation and interaction with the oocyte. (a) During capacitation, tyrosine phosphorylation appears first in the principal piece (PP) and then in the midpiece (MP) in about $20 \%$ of spermatozoa, whereas $15 \%$ of spermatozoa remain phosphorylated in the principal piece only. (b) After binding to the zona pellucida (ZP), tyrosine phosphorylation is stimulated in the midpiece, resulting in nearly $100 \%$ of spermatozoa phosphorylated in the whole flagellum. (c) After binding to the oolemma, tyrosine phosphorylation is also stimulated in the midpiece. Soon after fusion, the spermatozoa undergoing decondensation present tyrosine phosphorylation in the whole flagellum or restricted to the principal piece. Tyrosine phosphorylation limited to the principal piece in fused spermatozoa may be explained by a process of dephosphorylation of the flagellum after fusion, culminating in the dephosphorylation of the entire flagellum.

different sperm compartments during capacitation and interaction of spermatozoa with the oocyte.

\section{Protein tyrosine phosphorylation in the flagellum}

It is necessary to differentiate the localization of the tyrosine phosphorylated proteins in spermatozoa to understand the link between the different phosphorylated proteins and the corresponding regulated sperm function. Except in boars (Petrunkina et al., 2001; Tardif et al., 2001), the flagellum appears to be the principal sperm compartment presenting tyrosine phosphorylated proteins. Immunocytochemistry has been used to localize tyrosine phosphorylated proteins to the flagellum in human ( $\mathrm{Naz}$ et al., 1991; Carrera et al., 1996; Leclerc et al., 1997), monkey (Mahony and Gwathmey, 1999), hamster (Si and Okuno, 1999), rat (Lewis and Aitken, 2001), and mouse (Urner et al., 2001a) spermatozoa.

\section{Protein tyrosine phosphorylation during capacitation and interaction with the oocyte}

Protein tyrosine phosphorylation increases in spermatozoa during capacitation in a number of species including mice, humans, cattle, pigs, hamsters and cats (Leyton and Saling, 1989; Visconti et al., 1995a; Leclerc et al., 1997; Galantino-Homer et al., 1997; Kalab et al., 1998; Pukazhenthi et al., 1998; Si and Okuno, 1999). Protein tyrosine phosphorylation appears to be a necessary prerequisite for a spermatozoon to fertilize an egg (Visconti et al., 1995a; Urner et al., 2001).

Immunofluorescence was used to localize tyrosine phosphorylated proteins to the flagellum during capacitation, zona pellucida binding, oolemma binding and gamete fusion (Urner et al., 2001). A summary of the changes in tyrosine phosphorylation in the mouse flagellum (Fig. 1) shows that the proportion of spermatozoa presenting phosphorylated proteins in the whole flagellum increases with capacitation, and phosphorylation in the principal piece is a prerequisite for phosphorylation in the midpiece (Fig. 1a). A similar increase has been observed in human spermatozoa but was restricted to the principal piece (Sakkas et al., in press). In both mice and men, approximately $50 \%$ of spermatozoa remain non-phosphorylated after capacitation, indicating that there are subpopulations of spermatozoa that exhibit a different susceptibility to undergo tyrosine phosphorylation. The possibility that the phosphorylation-positive spermatozoa have a competitive advantage to fertilize an egg remains to be determined. This finding also has implications for certain types of infertility in males, as the proportion of spermatozoa in an ejaculate that are unable to undergo the correct sequence of phosphorylation to achieve capacitation may render them unable to fertilize an egg.

Although tyrosine phosphorylation during capacitation is not required for spermatozoa to bind to the zona pellucida in an in vitro system (Pukazhenthi et al., 1998), most mouse spermatozoa binding to the zona pellucida display a tyrosine phosphorylated principal piece, indicating that this pattern of phosphorylation is 
beneficial for binding. Binding to the zona pellucida maintains the principal piece phosphorylation and promotes tyrosine phosphorylation in the midpiece (Fig. 1b). Similarly, most of the spermatozoa bound to the oolemma display a tyrosine phosphorylated principal piece, whereas tyrosine phosphorylation in the midpiece is stimulated and maintained until fusion with the oocyte. Nearly all fused spermatozoa display a tyrosine phosphorylated flagellum that is subsequently dephosphorylated (Fig. 1c). In the absence of glucose, both sperm-oocyte fusion and tyrosine phosphorylation in the midpiece are inhibited, indicating that tyrosine phosphorylation in the whole flagellum is mandatory for fusion (Urner et al., 2001).

\section{Significance of protein tyrosine phosphorylation in the flagellum}

Tyrosine phosphorylated proteins in the flagellum are linked to sperm hyperactivated motility (Mahony and Gwathmey, 1999; Nassar et al., 1999; Si and Okuno, 1999), which is a motility pattern required for spermatozoa to penetrate the cumulus and the zona pellucida of the oocyte. Exposure of spermatozoa to drugs that increase intracellular cAMP enhances both hyperactivated motility and the proportion of spermatozoa presenting tyrosine phosphorylation in the flagellum in human (Nassar et al., 1999), monkey (Mahony and Gwathmey, 1999) and rat (Lewis and Aitken, 2001) spermatozoa. Glucose, which is required for hyperactivated motility (Cooper, 1984; Williams and Ford, 2001) and zona pellucida penetration (Urner and Sakkas, 1996a), promotes tyrosine phosphorylation in the flagellum in mouse spermatozoa (Urner et al., 2001).

Hyperactivated motility and the associated tyrosine phosphorylation in the flagellum are initiated during in vitro capacitation, and their maintenance in spermatozoa bound to the zona pellucida is of great importance (Stauss et al., 1995) and explains why the zona pellucida promotes the tyrosine phosphorylation of proteins in the flagellum. After penetration through the zona pellucida, the fertilizing spermatozoon binds to the oolemma and membrane fusion occurs subsequently between the equatorial segment of the sperm head and the microvilli of the oocyte. In addition, the sperm flagellum appears to fuse at multiple sites with the oocyte plasma membrane (Simerly et al., 1993). This process requires calcium (Yanagimachi, 1978) and glucose (Urner and Sakkas, 1996b; Redkar and Olds-Clarke, 1999), but the molecular events occurring during binding and fusion have yet to be elucidated. In contrast to the events during sperm penetration through the zona pellucida, hyperactivated motility is not required for spermatozoa to fuse with the oolemma. It is possible that the tyrosine phosphorylated proteins in the flagellum are linked to another sperm function important for both zona pellucida and oocyte penetration and that a common pathway is activated in spermatozoa interacting with the zona pellucida and the oolemma. The identification of the tyrosine phosphorylated proteins in the mouse midpiece would provide further insight into the specific role of this pattern of phosphorylation and how it influences membrane fusion occurring between the sperm equatorial segment and the oolemma.

\section{Tyrosine phosphorylated proteins in the flagellum}

In human spermatozoa, the A-kinase anchoring proteins (AKAPs) localized to the fibrous sheath AKAP82, its precursor pro-AKAP82, and FSP95 are the most prominent tyrosine phosphorylated proteins during capacitation (Carrera et al., 1996; Mandal et al., 1999), indicating that their affinity for the regulatory subunit of protein kinase $A$ or other molecules is regulated by their phosphorylation status. An 86 kDa calciumbinding and tyrosine phosphorylation-regulated protein (CABYR) is localized to the principal piece of human spermatozoa (Naaby-Hansen et al., 2002). Tyrosine phosphorylated CABYR acquires the capacity to bind calcium during capacitation and may therefore play a role in calcium sequestration and release in the principal piece, establishing a link between tyrosine phosphorylation and calcium, which is itself involved in regulating sperm-hyperactivated motility (Ho and Suarez, 2001). Although both AKAPs and CABYR are associated with the fibrous sheath, soluble proteins may also be associated with motility, as exemplified by the $55 \mathrm{kDa}$ tyrosine phosphorylated soluble protein linked to motility in bovine spermatozoa (Vijayaraghavan et al., 1997a).

\section{Protein tyrosine phosphorylation in the sperm head}

\section{Protein tyrosine phosphorylation during capacitation}

Tyrosine phosphorylation in the sperm head represents a minor pattern of phosphorylation in mouse spermatozoa. Non-capacitated spermatozoa are all non-phosphorylated, except for about 5-10\% which display protein tyrosine phosphorylation restricted to the acrosomal region (Leyton and Saling, 1989; Urner et al., 2001). In contrast to tyrosine phosphorylation in the flagellum, the proportion of spermatozoa displaying a tyrosine-phosphorylated acrosome does not increase with capacitation (Urner et al., 2001).

The only tyrosine-phosphorylated protein detected by western analysis in non-capacitated mouse spermatozoa is hexokinase, indicating that this enzyme is probably the tyrosine-phosphorylated protein localized to the acrosome in uncapacitated spermatozoa by immunofluorescence. In addition, both the phosphorylation status of hexokinase and the proportion of tyrosine phosphorylated acrosome are constant during capacitation. In mice, the germ-cell specific hexokinase (Kalab et al., 1994) is the major membrane tyrosine 
phosphorylated protein (116/95 kDa, reducing/nonreducing condition). Although hexokinase was localized to the head, midpiece and principal piece (Travis et al., 1998), the localization and the role of the phosphorylated form of the enzyme have not been determined.

The significance of tyrosine phosphorylation in the head during capacitation is unclear, but does not appear to be influenced by capacitation. In rats, tyrosine phosphorylation in the acrosomal region is characteristic of immature spermatozoa from the caput epididymidis (Lewis and Aitken, 2001). This pattern of phosphorylation decreases from 100 to $10-20 \%$ with maturation in the rat epididymis and the acrosome-positive spermatozoa that persist in the cauda epididymidis have been interpreted as representative of remaining immature spermatozoa.

\section{Protein tyrosine phosphorylation upon zona pellucida interaction}

A tyrosine phosphorylation step is necessary for the acrosome reaction induced by the zona pellucida, as inhibition of tyrosine phosphorylation prevents the acrosome reaction (Leyton et al., 1992; Pukazhenthi et al., 1998). In addition, exposure to progesterone, which induces the acrosome reaction, promotes an increase in tyrosine phosphorylation in the head of human spermatozoa (Tesarik et al., 1993). Solubilized zona pellucida has been used to observe an increase in tyrosine phosphorylation of a $95 \mathrm{kDa}$ protein in mouse spermatozoa (Leyton and Saling, 1989; Leyton et al., 1992). This protein, which is not hexokinase, localizes to the head surface in the acrosomal region and becomes tyrosine phosphorylated upon binding to the zona pellucida (Leyton et al., 1995). In humans, tyrosine phosphorylation of a protein of approximately $95 \mathrm{kDa}$ has also been observed after exposure to solubilized and recombinant ZP3 (Burks et al., 1995; Brewis et al., 1998). In contrast to the above studies, a high percentage of mouse spermatozoa bound to intact zona pellucida do not present any sign of tyrosine phosphorylation in the acrosomal region (Urner et al., 2001). This discrepancy might be explained if the tyrosine phosphorylated protein localized to the head is considered as a less abundant protein in which changes in phosphorylation are not detected easily by conventional immunofluorescence. In boars, the tyrosine phosphorylated plasma membrane proteins bound to the zona pellucida were found to be minor phosphorylated proteins compared with the tyrosine phosphorylated proteins of the whole sperm cells, even though they may still play a key role in spermzona pellucida interaction (Flesch et al., 1999, 2001).

\section{Protein tyrosine kinases in the sperm head}

The $95 \mathrm{kDa}$ sperm membrane protein that becomes tyrosine phosphorylated upon binding to the zona pellucida in mice possesses the characteristics of a receptor protein tyrosine kinase (Leyton et al., 1992). This protein displays intrinsic tyrosine kinase activity and contains phosphotyrosine that increases upon binding to ZP3; because of this property it has been referred to as zona receptor kinase (ZRK). A similar protein, Hu9, has been described in humans (Burks et al., 1995) but the Hu9 cDNA used in this study encoded for the human proto-oncogene c-mer instead of the zona receptor kinase, bringing into question the existence of a human homologue of mouse ZRK (Bork, 1996).

Receptor protein tyrosine kinases (RPTK) are transmembrane proteins presenting an extracellular binding domain and an intracellular tyrosine kinase domain. Upon extracellular ligand binding, kinase is activated and phosphorylates tyrosine residues on the RPTK itself (autophosphorylation) or on other proteins. After autophosphorylation of RPTK, intracellular signalling proteins such as phosphoinositide 3-kinase and phospholipase $\mathrm{C}_{\gamma}$ may be recruited to the phosphorylated domain of RPTK and activated. The targeting of a phospholipase $\mathrm{C} \gamma$ to the plasma membrane and its tyrosine phosphorylation-dependent activation in mouse spermatozoa (Tomes et al., 1996), and phosphoinositide 3-kinase activity operating downstream of tyrosine phosphorylation in human spermatozoa (Fisher et al., 1998) indirectly indicate that a receptor protein tyrosine kinase is operative in spermatozoa.

Non-receptor protein tyrosine kinases may also be present in the sperm head, as c-yes, which is a member of the nonreceptor Src family of tyrosine kinases, has been detected in the human sperm head (Leclerc and Goupil, 2002). The activity of the c-yes kinase depends on cAMP, indicating that tyrosine phosphorylation of proteins in the sperm head results from the cross-talk between the cAMP pathway and tyrosine-kinases, as it does in the flagellum.

\section{Regulation of protein tyrosine phosphorylation}

Tyrosine phosphorylation occurs spontaneously in spermatozoa in vitro, provided they are diluted in culture medium supporting capacitation (Urner et al., 2001) and freed of seminal plasma (Tomes et al., 1998). In vivo, the appearance of capacitation-associated tyrosine phosphorylation is probably regulated by sperm binding to oviductal epithelial cells before fertilization (Petrunkina et al., 2001). The oocyte itself triggers changes in tyrosine phosphorylation that are linked to the acrosome reaction and other functions related to gamete fusion. The sequence of events responsible for capacitation, including removal of cholesterol from the sperm plasma membrane, increase in bicarbonate uptake, activation of adenylate cyclase, and the crosstalk between the cAMP pathway and protein tyrosine phosphorylation have been described in reviews by Visconti and Kopf (1998) and Visconti et al. (2002). This 
section describes recent contributions to our knowledge on protein kinases and glucose metabolism in regulating tyrosine phosphorylation.

\section{cAMP-dependent protein kinase A}

The cAMP-dependent protein kinase A (PKA) plays a central role in sperm capacitation, motility and the acrosome reaction (Bielfeld et al., 1994; Visconti et al., 1995b; Harrison et al., 2000; Skalhegg et al., 2002). PKA regulates protein tyrosine phosphorylation in spermatozoa by either direct or indirect effects on tyrosine kinase or phosphatases (Visconti et al., 2002). PKA is a tetrameric enzyme composed of two regulatory and two catalytic subunits. Two isoforms of the regulatory subunit (RI and RII) are present in spermatozoa (Vijayaraghavan et al., 1997b; Visconti et al., 1997). The activity of PKA is dependent on the amount of cAMP, which is in turn regulated by adenylate cyclase and phosphodiesterase. The binding of cAMP to the regulatory subunit promotes the dissociation and activation of the catalytic subunits that catalyse phosphorylation on serine/threonine residues.

PKA is anchored to specific sperm compartments through binding of its regulatory subunit to the AKAPs. In general, AKAPs are implicated in the regulation of protein phosphorylation by the tethering of protein kinases and phosphatases in close proximity to their target proteins within specific cell compartments. Different types of AKAP have been found in spermatozoa (for review, see Moss and Gerton, 2001). AKAP82 (Carrera et al., 1994; Johnson et al., 1997) and FSP95 (Mandal et al., 1999) are both localized to the fibrous sheath of the principal piece and anchor the RII subunit of PKA. AKAP110, localized to the principal piece and the acrosomal region (Vijayaraghavan et al., 1999), presents unique characteristics in spermatozoa. In addition to binding to the regulatory subunit of PKA, the sperm AKAP110 interacts with other signalling proteins (such as ropporin) that may regulate motility independently of PKA (Carr et al., 2001). Two other AKAPS have been identified in the midpiece: S-AKAP84, which is associated with mitochondria (Lin et al., 1995) and AKAP220, which is anchored to cytoskeletal structures and binds both the RII and RI subunits (Reinton et al., 2000). The localization of AKAPs in all sperm compartments indicates that they participate indirectly (through scaffolding of PKA or other signalling molecules) in the regulation of tyrosine phosphorylation in both the flagellum and the sperm head. In addition to participating in the regulation of protein phosphorylation in spermatozoa, AKAPs are themselves phosphorylated on tyrosine residues in humans (AKAP82, FSP95; Carrera et al., 1996; Mandal et al., 1999) and on serine/threonine residues in mice (AKAP 82; Johnson et al., 1997) during capacitation, indicating that their functions are modified by their phosphorylation status.

\section{Mitogen-activated protein kinases}

The mitogen-activated protein kinases (MAPK), also known as extracellular signal-regulated kinases (ERK), are serine/threonine kinases involved in signal transduction of several extracellular messengers. Their activity is regulated by a phosphorylation cascade initially triggered by the GTP-binding protein Ras or protein kinase C. MAPK-kinase-kinase (Raf), which is the first enzyme of this cascade, phosphorylates MAPK-kinases (MEK) on serine/threonine, which in turn phosphorylates MAPK on threonine and tyrosine residues. MAPK requires phosphorylation on both threonine and tyrosine to become active.

The MAPK pathway has been identified in spermatozoa and appears to play a role in capacitation (de Lamirande and Gagnon, 2002) and the acrosome reaction (Luconi et al., 1998; du Plessis et al., 2001). The zona pellucida- and lysophosphatidylcholineinduced acrosome reactions are prevented when MAPK activity is inhibited (du Plessis et al., 2001; de Lamirande and Gagnon, 2002). Progesterone promotes the phosphorylation-activation of the MAPK isoform ERK2 in human spermatozoa and its redistribution from the post-acrosomal region to the equatorial segment (Luconi et al., 1998). The MAPK isoform ERK2 (Luconi et al., 1998), the adaptor protein Shc (Morte et al., 1998) and Ras (Naz et al., 1992) have all been localized to the sperm head, indicating that this pathway is required for controlling protein phosphorylation in the sperm head.

Although the proteins phosphorylated by MAPK in spermatozoa remain to be identified, de Lamirande and Gagnon (2002) reported a 75 and $80 \mathrm{kDa}$ protein presenting a phosphorylated serine/threonineproline motif recognized by the MPM-2 antibody, which is consistent with a MAPK-phosphorylated epitope. MPM2-reactive proteins in the 77-85 $\mathrm{kDa}$ range have been detected in the rabbit sperm midpiece and connective piece in different species (Pinto-Correia et al., 1994; Long et al., 1997) and may be components of the centrosome in the connecting piece (Simerly et al., 1999). Therefore, centrosomal proteins may be considered as candidate substrates of MAPK in spermatozoa. In addition, MAPK may phosphorylate proteins that influence protein tyrosine phosphorylation indirectly, as inhibition of MAPK prevents protein tyrosine phosphorylation associated with capacitation (de Lamirande and Gagnon, 2002).

\section{Protein phosphatases}

Tyrosine phosphorylation is stimulated indirectly by activation of phosphorylation on serine/threonine (by PKA or MAPK) and, therefore, it is conceivable that dephosphorylation by phosphatases of these residues inhibits tyrosine phosphorylation. Serine/threoninespecific protein phosphatase (PP) activities have been 
identified in spermatozoa from different species and are involved in the regulation of sperm motility. The isoform PP1 $\gamma 2$ has been detected in primate spermatozoa (Smith et al., 1996). PP1 colocalizes with AKAP220 to cytoskeleton structures, indicating that it is anchored via AKAP220 in the midpiece (Reinton et al., 2000). The activity of PP1 is regulated by the PP1 inhibitor 2 (I2) and glycogen synthase kinase 3 (GSK-3), which are both present in spermatozoa (Smith et al., 1996). PP1 is inactive when bound to non-phosphorylated I2 and becomes activated when $\mathrm{I} 2$ is phosphorylated by GSK-3, implying that PP1-dependent dephosphorylation of sperm proteins parallels GSK-3 activity, which may be itself regulated by phosphorylation, as has been reported in somatic cells. The calcium/calmodulindependent phosphatase calcineurin (PP2B) has been detected in dog spermatozoa (Tash et al., 1988) and appears to participate in dephosphorylation of tyrosine phosphorylated substrates in human spermatozoa (Carrera et al., 1996).

\section{Glucose metabolism}

Glucose participates in the regulation of protein tyrosine phosphorylation during capacitation and gamete interaction in vitro. In the absence of glucose, tyrosine phosphorylation in the principal piece is delayed and phosphorylation in the midpiece is inhibited as a consequence of this delay or because of a local inhibition in the midpiece. The absence of glucose and the delayed tyrosine phosphorylation during capacitation do not affect fertilization, as long as glucose is added during gamete mixing. However, the absence of glucose during gamete interaction prevents fertilization, indicating that a precise timing in the induction of tyrosine phosphorylation once the spermatozoa bind to the oocyte is critical for successful fertilization. The observation that high and low concentrations of glucose are necessary to support gamete fusion (Urner and Sakkas, 1996b) and protein tyrosine phosphorylation during capacitation (Travis et al., 2001a), respectively, emphasizes the predominant role of glucose during gamete interaction.

The utilization of glucose depends on its availability in the environment, its uptake by specific transporters and its intracellular metabolism. Spermatozoa are compartmentalized with regard to glucose utilization, indicating a specific regulation in the different sperm domains during the course of fertilization (Fig. 2). The high affinity glucose transporter GLUT3 was localized mainly to the midpiece (Angulo et al., 1998; Rigau et al., 2002) and the recently identified GLUT8 has been localized to the acrosomal region (Schürmann et al., 2002).

The glucose metabolic pathways detected in mouse spermatozoa are glycolysis and pentose phosphate pathways (Urner and Sakkas, 1999). Hexokinase, which phosphorylates glucose to glucose 6-phosphate, is present in the sperm head, midpiece and principal piece (Travis et al., 1998). Glycolysis is associated predominantly with the sperm principal piece (Westhoff and Kamp, 1997; Bunch et al., 1998; Welch et al., 2000) and the pentose phosphate pathway with the sperm midpiece (Zimmermann and Geyer, 1981) and the sperm head (D. Sakkas, G. Leppens-Luisier and F. Urner, unpublished). The oxidative phosphorylation takes place in the mitochondria localized to the sperm midpiece.

Glucose has to be metabolized by spermatozoa for zona pellucida penetration, sperm-oocyte fusion and to ensure that tyrosine phosphorylation occurs during capacitation (Urner and Sakkas, 1996a,b; Travis et al., 2001). Glucose is provided to spermatozoa by the female reproductive tract fluid in vivo or by the culture medium in vitro, but studies have indicated that stores of glycogen are endogenous sources of glucose in spermatozoa (Ballester et al., 2000; Rigau et al., 2002), allowing spermatozoa to accommodate glucosefree conditions.

ATP produced by glycolysis or oxidative respiration is absolutely required for phosphorylation itself and cAMP synthesis. Although Travis et al. (2001a) postulated that the ATP produced by the glycolytic pathway was strictly required for protein tyrosine phosphorylation, the observation that NADPH can modulate protein tyrosine phosphorylation in human (Aitken et al., 1995; de Lamirande et al., 1998) and mouse spermatozoa (Urner et al., 2001) indicates that the pentose phosphate pathway is also involved. Delay rather than a total inhibition of tyrosine phosphorylation indicates that ATP and NADPH are generated by spermatozoa in the absence of glucose but to a lesser extent than in its presence. In the absence of glucose, the main sources of ATP are mitochondria in the sperm midpiece. Because the phosphorylcreatine shuttle transporting phosphate from the sperm midpiece to the principal piece is poorly developed in mammalian spermatozoa (Kamp et al., 1996), the principal piece may be partially deprived of ATP, explaining the delayed protein phosphorylation in this compartment. The maintenance of sperm motility in the absence of glucose indicates that ATP may be available to the dynein ATPases of the axoneme in the principal piece in the absence of metabolism through glycolysis. In contrast, delayed tyrosine phosphorylation in the midpiece is not the result of ATP deprivation but may be the consequence of low NADPH generation. In the absence of glucose, NADPH may be produced by alternate cytosolic pathways such as the malic enzyme or the NADP-dependent isocitrate dehydrogenase, which may not be as efficient as the pentose phosphate pathways in generating this nucleotide.

NADPH is involved in oxido-reductive reactions and is required for NADPH-dependent enzymes such as NADPH-oxidase and nitric oxide synthase. The influence of superoxide and hydrogen peroxide on sperm function and tyrosine phosphorylation (Aitken et al., 


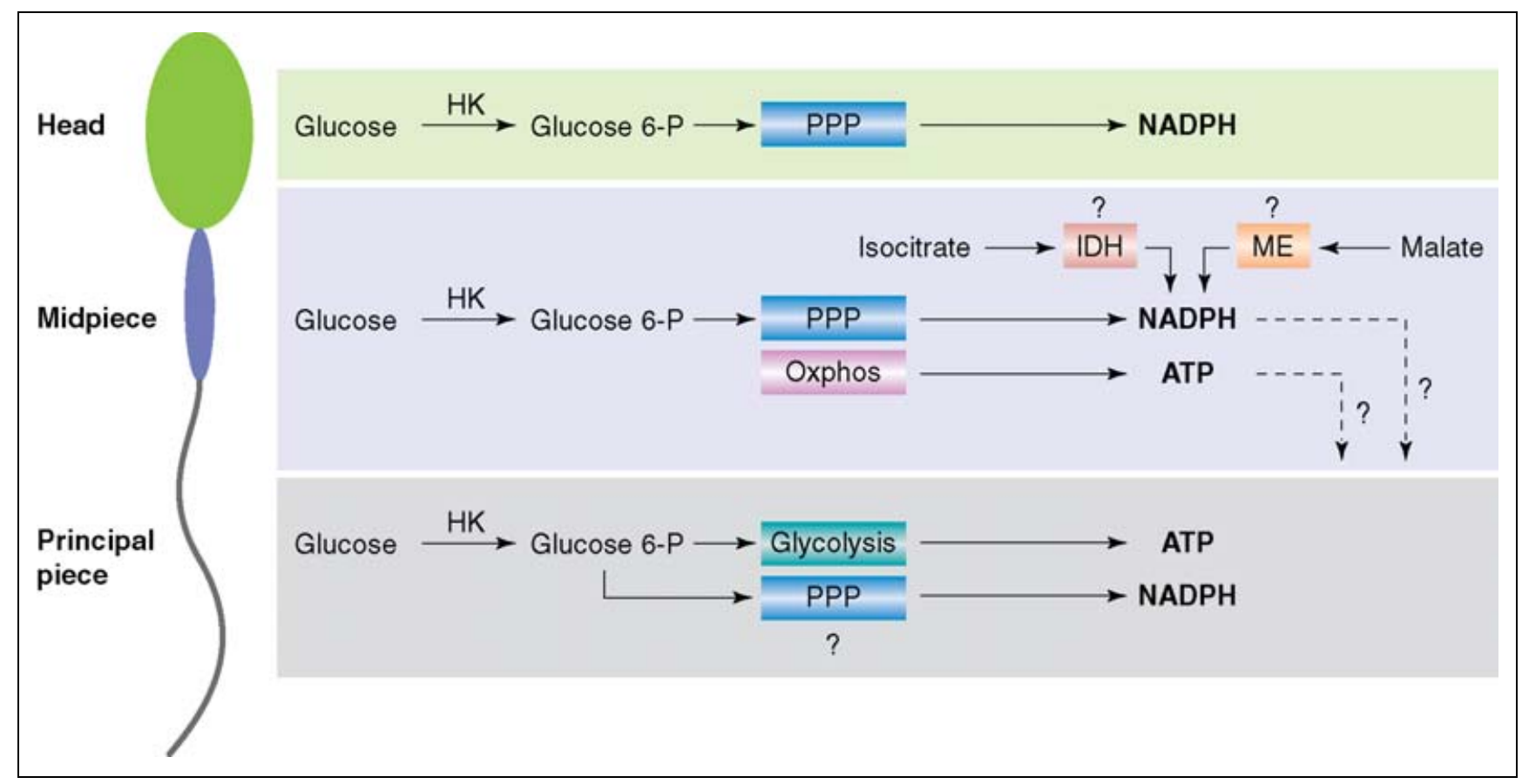

Fig. 2. Compartmentalization of glucose metabolism in the sperm flagellum. The uptake of glucose occurs through specific membrane transporters. In the presence of glucose, ATP is generated through glycolysis in the principal piece and through oxidative phosphorylation (Oxphos) in the midpiece. NADPH is generated by the pentose phosphate pathway (PPP) in the midpiece and the sperm head. Availability of NADPH in the principal piece through transport from the midpiece or local PPP has not been demonstrated. In the absence of glucose, ATP generated from oxidative phosphorylation may be transported to the principal piece. NADPH may be produced in the midpiece by the cytosolic NADP-dependent isocitrate dehydrogenase (IDH) or malic enzyme (ME). HK: hexokinase.

1995; Leclerc et al., 1997) indicates that an NADPHoxidase is involved, although superoxide may also be produced by the mitochondrial electron transport chain (Vernet et al., 2001). Nitric oxide synthase, catalysing nitric oxide synthesis, is present in mouse and human spermatozoa (Herrero et al., 1997; Revelli et al., 1999) and plays a physiological role in sperm function (Zini et al., 1995; Francavilla et al., 2000) as well as in the regulation of tyrosine phosphorylation (Herrero et al., 1999). On account of its calcium and NADPH requirement, it is possible that nitric oxide synthase links glucose metabolism and the regulation of tyrosine phosphorylation in spermatozoa.

\section{Conclusion}

The passage of spermatozoa in the male and female reproductive tract requires precise timing so as to synchronize the fertilization competence of the spermatozoon with ovulation. A specific sequence of events must occur to achieve the correct priming of the fertilizing spermatozoa. Several studies have indicated that the spermatozoa achieve this by a specific sequence of phosphorylation in the flagellum. The role of phosphorylation in the sperm head remains unclear. Molecules involved in the sequence of phosphorylation are more likely to arise from the milieu surrounding the spermatozoa, but their uptake and processing are probably regulated in different ways at specific steps within the female genital tract and during penetration of the egg vestments. The sperm functions known to be associated with changes in protein tyrosine phosphorylation are capacitation, hyperactivated motility, acrosome reaction and spermoocyte fusion. These events are regulated by the activation of compartmentalized intracellular signalling systems. One such signalling mechanism may be related to the recently reported effects of caveolins on the sperm membrane (Travis et al., 2001b). The metabolic products of glucose (ATP and NADPH) appear to participate in these signalling pathways by supporting a precise onset of tyrosine phosphorylation in the sperm flagellum leading to successful fertilization. A greater understanding of the compartmentalized signals controlling phosphorylation in spermatozoa will provide insight into how spermatozoa are primed to enter the egg and may improve understanding of aspects of human infertility.

This work was supported by the Fonds National Suisse de la Recherche Scientifique.

\section{References}

Key references are identified by asterisks

*Aitken RJ, Paterson M, Fisher H, Buckingham DW and van Duin M (1995) Redox regulation of tyrosine phosphorylation in human spermatozoa 
and its role in the control of human sperm function Journal of Cell Science 108 2017-2025

Angulo C, Rauch MC, Droppelmann A, Reyes AM, Slebe JC, DelgadoLopez F, Guaiquil VH, Vera JC and Concha IL (1998) Hexose transporter expression and function in mammalian spermatozoa: cellular localization and transport of hexoses and vitamin C Journal of Cellular Biochemistry 71 189-203

Ballester J, Fernandez-Novell JM, Rutllant J, Garcia-Rocha M, Jesus Palomo M, Mogas T, Pena A, Rigau T, Guinovart JJ and Rodriguez-Gil JE (2000) Evidence for a functional glycogen metabolism in mature mammalian spermatozoa Molecular Reproduction and Development 56 207-219

Bielfeld P, Faridi A, Zaneveld LJD and De Jonge CJ (1994) The zona pellucida-induced acrosome reaction of human spermatozoa is mediated by protein kinases Fertility and Sterility 61 536-541

Bork P (1996) Sperm-egg binding protein or proto-oncogene? Science 27 $11431-11432$

Brewis IA, Clayton R, Browes CE, Martin M, Barratt CLR, Hornby DP and Moore HDM (1998) Tyrosine phosphorylation of a 95 kDa protein and induction of the acrosome reaction in human spermatozoa by recombinant human zona pellucida glycoprotein 3 Molecular Human Reproduction 4 1136-1144

Bunch DO, Welch JE, Magyar PL, Eddy EM and O'Brien DA (1998) Glyceraldehyde 3-phosphate dehydrogenase-S protein distribution during mouse spermatogenesis Biology of Reproduction 58834 841

Burks DJ, Carballada R, Moore HD and Saling PM (1995) Interaction of a tyrosine kinase from human sperm with the zona pellucida at fertilization Science 269 83-86

Carr DW, Fujita A, Stentz CL, Liberty GA, Olson GE and Narumiya S (2001) Identification of sperm-specific proteins that interact with Akinase anchoring proteins in a manner similar to the type II regulatory subunit of PKA Journal of Biological Chemistry 27617 332-17 338

Carrera A, Gerton GL and Moss SB (1994) The major fibrous sheath polypeptide of mouse sperm: structural and functional similarities to the A-kinase anchoring proteins Developmental Biology 165 $272-284$

Carrera A, Moos J, Ning XP, Gerton GL, Tesarik J, Kopf GS and Moss SB (1996) Regulation of protein tyrosine phosphorylation in human sperm by a calcium/calmodulin-dependent mechanism: identification of A kinase anchor proteins as major substrates for tyrosine phosphorylation Developmental Biology 180 284-296

Cooper TG (1984) The onset and maintenance of hyperactivated motility of spermatozoa from the mouse Gamete Research 9 55-74

De Lamirande E and Gagnon C (2002) The extracellular signal-regulated kinase(ERK) pathway is involved in human sperm function and modulated by the superoxide anion Molecular Human Reproduction 8 124-135

De Lamirande E, Harakat A and Gagnon C (1998) Human sperm capacitation induced by biological fluids and progesterone, but not by $\mathrm{NADH}$ or $\mathrm{NADPH}$, is associated with the production of superoxide anion Journal of Andrology 19 215-225

Du Plessis SS, Page C and Franken DR (2001) The zona pellucida-induced acrosome reaction of human spermatozoa involves extracellular signalregulated kinase activation Andrologia 33 337-342

Fisher HM, Brewis IA, Barratt CLR, Cooke ID and Moore HDM (1998) Phosphoinositide 3-kinase is involved in the induction of the human sperm acrosome reaction downstream of tyrosine phosphorylation Molecular Human Reproduction 4 849-855

Flesch FM, Colenbrander B, van Golde LM and Gadella BM (1999) Capacitation induces tyrosine phosphorylation of proteins in the boar sperm plasma membrane Biochemical and Biophysical Research Communications 262 787-792

Flesch FM, Wijnand $\mathrm{E}$, van de Lest $\mathrm{CH}$, Colenbrander B, van Golde LM and Gadella BM (2001) Capacitation dependent activation of tyrosine phosphorylation generates two sperm head plasma membrane proteins with high primary binding affinity for the zona pellucida Molecular Reproduction and Development 60 107-115

Francavilla F, Santucci R, Macerola B, Ruvolo G and Romani R (2000) Nitric oxide synthase inhibition in human sperm affects sperm-oocyte fusion but not zona pellucida binding Biology of Reproduction 63 $425-429$

Galantino-Homer HL, Visconti PE and Kopf GS (1997) Regulation of protein tyrosine phosphorylation during bovine sperm capacitation by a cyclic adenosine 3'5'-monophosphate-dependent pathway Biology of Reproduction 56 707-719

Harrison DA, Carr DW and Meizel S (2000) Involvement of protein kinase $\mathrm{A}$ and $\mathrm{A}$ kinase anchoring protein in the progesterone-initiated human sperm acrosome reaction Biology of Reproduction 62811 820

Herrero MB, Goin JC, Boquet M, Canteros MG, Franchi AM, Martinez SP, Polak JM, Viggiano JM and Gimeno MAF (1997) The nitric oxide synthase of mouse spermatozoa FEBS Letters 411 39-42

*Herrero MB, de Lamirande E and Gagnon C (1999) Nitric oxide regulates human sperm capacitation and protein tyrosine phosphorylation in vitro. Biology of Reproduction 61 575-581

Ho HC and Suarez SS (2001) Hyperactivation of mammalian spermatozoa: function and regulation Reproduction 122 519-526

Johnson LR, Foster JA, Haig-Ladewig L, VanScoy H, Rubin CS, Moss SB and Gerton GL (1997) Assembly of AKAP82, a protein kinase A anchor protein, into the fibrous sheath of mouse sperm Developmental Biology 192 340-350

Kalab P, Visconti P, Leclerc P and Kopf GS (1994) p95, the major phosphotyrosine containing protein in mouse spermatozoa, is a hexokinase with unique properties Journal of Biological Chemistry 2693810 3817

Kalab P, Peknicova J, Geussova G and Moss J (1998) Regulation of protein tyrosine phosphorylation in boar sperm through a cAMP-dependent pathway Molecular Reproduction and Development 51 304-314

Kamp G, Büsselmann G and Lauterwein J (1996) Spermatozoa: models for studying regulatory aspects of energy metabolism Experientia 52 487494

Leclerc P and S Goupil (2002) Regulation of the human sperm tyrosine kinase c-yes. Activation by cyclic adenosine $3^{\prime}, 5^{\prime}$-monophosphate and inhibition by $\mathrm{Ca}^{2+}$ Biology of Reproduction 67 301-307

Leclerc P, de Lamirande E and Gagnon C (1997) Regulation of protein-tyrosine phosphorylation and human sperm capacitation by reactive oxygen derivatives Free Radicals in Biological Medicine 22 643-656

Lewis B and Aitken RJ (2001) Impact of epididymal maturation on the tyrosine phosphorylation patterns exhibited by rat spermatozoa Biology of Reproduction 64 1545-1556

Leyton L and Saling P (1989) 95 kDa sperm proteins bind ZP3 and serve as tyrosine kinase substrates in response to zona binding Cell 571123 1130

*Leyton L, LeGuen P, Bunch D and Saling P (1992) Regulation of mouse gamete interaction by a sperm tyrosine kinase Proceedings National Academy of Sciences USA $8911692-11695$

Leyton L, Tomes C and Saling P (1995) LL95 monoclonal antibody mimics functional effects of ZP3 on mouse sperm: evidence that the antigen recognized is not hexokinase Molecular Reproduction and Development 42 347-358

Lin R-Y, Moss SB and Rubin CS (1995) Characterization of S-AKAP84, a novel developmentally regulated $\mathrm{A}$ kinase anchor protein of male germ cells Journal of Biological Chemistry 27027 804-27 811

Long CR, Duncan RP and Robl JM (1997) Isolation and characterization of MPM-2-reactive sperm proteins: homology to components of the outer dense fibers and segmented columns Biology of Reproduction $\mathbf{5 7}$ 246-254

Luconi M, Krausz C, Barni T, Vanelli GB, Forti G and Baldi E (1998) Progesterone stimulates p42 extracellular signal-regulated kinase (p42 erk) in human spermatozoa Molecular Human Reproduction 4 251-258

Mahony MC and Gwathmey TY (1999) Protein tyrosine phosphorylation during hyperactivated motility of cynomolgus monkey (Macaca fascicularis) spermatozoa Biology of Reproduction 60 1239-1243

Mandal A, Naaby-Hansen S, Wolkowicz MJ et al. (1999) FSP95, a testis-specific 95-kilodalton fibrous sheath antigen that undergoes tyrosine phosphorylation in capacitated human spermatozoa Biology of Reproduction 61 1184-1197 
Morte C, Iborra A and Martinez P (1998) Phosphorylation of Shc proteins in human sperm in response to capacitation and progesterone treatment Molecular Reproduction and Development 50 113-120

Moss SB and Gerton GL (2001) A-kinase anchor proteins in endocrine systems and reproduction Trends in Endocrinology and Metabolism 12 434-440

Naaby-Hansen S, Mandal A, Wolkowicz MJ et al. (2002) CABYR, a novel calcium-binding tyrosine phosphorylation-regulated fibrous sheath protein involved in capacitation Developmental Biology 242 236-254

Nassar A, Mahony M, Morshedi M, Lin MH, Srisombut C and Oehninger S (1999) Modulation of sperm tail protein tyrosine phosphorylation by pentoxifylline and its correlation with hyperactivated motility Fertility and Sterility 71 919-923

Naz RK, Ahmad K and Kumar R (1991) Role of membrane phosphotyrosine proteins in human spermatozoal function Journal of Cell Science $\mathbf{9 9}$ 157-165

Naz RK, Ahmad K and Kaplan P (1992) Expression and function of rasproto-oncogene proteins in human sperm cells Journal of Cell Science 102 487-494

Petrunkina AM, Friedrich J, Drommer W, Bicker G, Waberski D and TöpferPetersen E (2001) Kinetic characterization of the changes in protein tyrosine phosphorylation of membranes, cytosolic $\mathrm{Ca}^{2+}$ concentration and viability in boar sperm populations selected by binding to oviductal epithelial cells Reproduction 122 469-480

Pinto-Correia C, Poccia DL, Chang T and Robl JM (1994) Dephosphorylation of sperm midpiece antigens initiates aster formation in rabbit oocytes Proceedings National Academy of Sciences USA 91 7894-7898

Pukazhenthi BS, Wildt DE, Ottinger MA and Howard J (1998) Inhibition of domestic cat spermatozoa acrosome reaction and zona pellucida penetration by tyrosine kinase inhibitors Molecular Reproduction and Development 49 48-57

Redkar AA and Olds-Clarke PJ (1999) An improved mouse sperm plasmalemma binding assay: studies on characteristics of sperm binding in medium with or without glucose Journal of Andrology 20 500-508

Reinton N, Collas P, Haugen TB, Skalhegg BS, Hansson V, Jahnsen T and Tasken K (2000) Localization of a novel human A-kinase-anchoring protein, hAKAP220, during spermatogenesis Developmental Biology 223 194-204

Revelli A, Soldati G, Costamagnana C, Pellerey O, Aldieri E, Massobrio M, Bosia A and Ghigo D (1999) Follicular fluid proteins stimulate nitric oxide (NO) synthesis in human sperm: a possible role for $\mathrm{NO}$ in acrosomal reaction Journal of Cell Physiology 178 85-92

Rigau T, Rivera M, Palomo MJ et al. (2002) Differential effect of glucose and fructose on hexose metabolism in dog spermatozoa Reproduction 123 579-591

Sakkas D, Leppens-Luisier G, Lucas H, Chardonnens D, Campana A, Franken D and Urner F (2003) Localization of tyrosine phosphorylated proteins in human sperm and relation to capacitation and zona pellucida binding Biology of Reproduction $\mathbf{6 8}$ (in press)

Schürmann A, Axer H, Scheepers A, Doege H and Joost HG (2002) The glucose transport facilitator GLUT8 is predominantly associated with the acrosomal region of mature spermatozoa Cell and Tissue Research 307 237-242

Si Y and Okuno M (1999) Role of tyrosine phosphorylation of flagellar proteins in hamster sperm hyperactivation Biology of Reproduction $\mathbf{6 1}$ 240-246

Simerly CR, Hecht NB, Goldberg E and Schatten G (1993) Tracing the incorporation of the sperm tail in the mouse zygote and early embryo using an anti-testicular $\alpha$-tubulin antibody Developmental Biology 158 536-548

Simerly C, Zoran SS, Payne C, Dominko T, Sutovsky P, Navara CS, Salisbury JL and Schatten G (1999) Biparental inheritance of $\boldsymbol{\gamma}$-tubullin during human fertilization: molecular reconstitution of functional zygotic centrosomes in inseminated human oocytes and in cell-free extracts nucleated by human sperm Molecular Biology of the Cell 10 29552969

Skalhegg BS, Huang Y, Su T, Idzerda RL, McNight GS and Burton KA (2002) Mutation of the $\mathrm{C} \alpha$ subunit of PKA leads to growth retardation and sperm dysfunction Molecular Endocrinology 16 630-639
Smith GD, Wolf DP, Trautman KC, da Cruz e Silva EF, Greengard P and Vijayaraghavan S (1996) Primate sperm contain protein phosphatase 1, a biochemical mediator of motility Biology of Reproduction 54719 727

Stauss CR, Votta TJ and Suarez SS (1995) Sperm motility hyperactivation facilitates penetration of the hamster zona pellucida Biology of Reproduction 53 1280-1285

Tardif S, Dube C, Chevalier S and Bailey JL (2001) Capacitation is associated with tyrosine phosphorylation and tyrosine like activity of pig sperm proteins Biology of Reproduction 65 784-792

Tash JS, Krinls M, Patel J, Means RL, Klee CB and Means AR (1988) Identification, characterization, and functional correlations of calmodulin-dependent protein phosphatase in sperm Journal of Cell Science 106 1625-1633

Tesarik J, Moos J and Mendoza C (1993) Stimulation of protein tyrosine phosphorylation by a progesterone receptor on the cell surface of human sperm Endocrinology 133 328-335

Tomes CN, McMaster CR and Saling P (1996) Activation of mouse sperm phosphatidylinositol-4,5 biphosphate-phospholipase $\mathrm{C}$ by zona pellucida is modulated by tyrosine phosphorylation Molecular Reproduction and Development 43 196-204

Tomes CN, Carballada R, Moses DF, Katz DF and Saling P (1998) Treatment of human spermatozoa with seminal plasma inhibits protein tyrosine phosphorylation Molecular Human Reproduction 4 17-25

Travis AJ, Foster JA, Rosenbaum NA, Visconti PE, Gerton GL, Kopf GS and Moss SB (1998) Targeting of a germ cell-specific type 1 hexokinase lacking a porin-binding domain to the mitochondria as well as to the head and fibrous sheath of murine spermatozoa Molecular Biology of the Cell 9 263-276

*Travis AJ, Jorgez $\mathrm{CJ}$, Merdiushev T, Jones BH, Dess DM, Diaz-Cueto L, Storey BT, Kopf GS and Moss SB (2001a) Functional relationships between capacitation-dependent cell signaling and compartmentalized metabolic pathways in murine spermatozoa Journal of Biological Chemistry 276 7630-7636

Travis AJ, Merdiushev T, Vargas LA et al. (2001b) Expression and localization of caveolin-1, and the presence of membrane rafts, in mouse and guinea pig spermatozoa Developmental Biology 15 599-610

Urner F and Sakkas D (1996a) Glucose is not essential for the occurrence of sperm binding and zona pellucida-induced acrosome reaction in the mouse International Journal of Andrology 19 91-96

Urner F and Sakkas D (1996b) Glucose participates in sperm-oocyte fusion in the mouse Biology of Reproduction 55 917-922

Urner F and Sakkas D (1999) A possible role for the pentose phosphate pathway of spermatozoa in gamete fusion in the mouse Biology of Reproduction 60 733-739

*Urner F, Leppens-Luisier G and Sakkas D (2001) Protein tyrosine phosphorylation in sperm during gamete interaction in the mouse: the influence of glucose Biology of Reproduction 64 1350-1357

Vernet P, Fulton N, Wallace C and Aitken RJ (2001) Analysis of reactive oxygen species generating systems in rat epididymal spermatozoa Biology of Reproduction 65 1102-1113

Vijayaraghavan S, Trautman KD, Goueli SA and Carr DW (1997a) A tyrosine-phosphorylated 55-kilodalton motility-associated bovine sperm protein is regulated by cyclic adenosine $3^{\prime}, 5^{\prime}$-monophosphates and calcium Biology of Reproduction 56 1450-1457

Vijayaraghavan S, Olson GE, NagDas S, Winfrey VP and Carr DW (1997b) Subcellular localization of the regulatory subunits of cyclic adenosine 3', 5'-monophosphate-dependent protein kinase in bovine spermatozoa Biology of Reproduction 57 1517-1523

Vijayaraghavan S, Liberty GA, Mohan J, Winfrey VP, Olson GE and Carr DW (1999) Isolation and molecular characterization of AKAP110, a novel, sperm-specific protein kinase A-anchoring protein Molecular Endocrinology 13 705-717

*Visconti PE and Kopf GS (1998) Regulation of protein phosphorylation during sperm capacitation Biology of Reproduction 59 1-6

Visconti PE, Bailey JL, Moore GD, Pan D, Olds-Clarke P and Kopf GS (1995a) Capacitation of mouse spermatozoa. I. Correlation between the capacitation state and protein tyrosine phosphorylation Development 121 1129-1137 
Visconti PE, Moore GD, Bailey JL, Leclerc P, Connors SA, Pan D, OldsClarke P and Kopf GS (1995b) Capacitation of mouse spermatozoa. II. Protein tyrosine phosphorylation and capacitation are regulated by a cAMP-dependent pathway Development 121 1139-1150

Visconti PE, Johnson LR, Oyaski M, Fornes M, Moss SB, Gerton GL and Kopf GS (1997) Regulation, localization, and anchoring of protein kinase A subunits during mouse sperm capacitation Developmental Biology 192 351-363

Visconti PE, Westbrook VA, Chertihin O, Demarco I, Sleight S and Diekman AB (2002) Novel signaling pathways involved in sperm acquisition of fertilizing capacity Journal of Reproductive Immunology 53 133-150

Welch JE, Brown PL, O'Brien DA, Magyar PL, Bunch DO, Mori C and Eddy EM (2000) Human glyceraldehyde 3-phosphate dehydrogenase-2 gene is expressed specifically in spermatogenic cells Journal of Andrology 21 $328-338$
Westhoff D and Kamp G (1997) Glyceraldehyde 3-phosphate dehydrogenase is bound to the fibrous sheath of mammalian spermatozoa Journal of Cell Science 110 1821-1829

Williams AC and Ford WC (2001) The role of glucose in supporting motility and capacitation in human spermatozoa Journal of Andrology 22 680-695

Yanagimachi R (1978) Calcium requirement for sperm-egg fusion in mammals Biology of Reproduction 19 949-958

Zimmermann N and Geyer G (1981) A polyacrylamide gel method for the cytochemical demonstration of glucose 6-phosphate dehydrogenase activity in mouse sperm Acta Histochemica 68 227-230

Zini A, de Lamirande E and Gagnon C (1995) Low levels of nitric oxide promote human sperm capacitation in vitro. Journal of Andrology 16 424-431 